\title{
Staring RF Signal Processing Challenges
}

\author{
Linda J. Moore* \\ Uttam K. Majumder*
}

\author{
Jason T. Parker* \\ Michael J. Minardi*
}

\author{
LeRoy A. Gorham* \\ Steven M. Scarborough*
}

\begin{abstract}
Traditionally, distinct radar modes have been employed to accomplish specific tasks such as imaging an area of interest, or detecting and tracking moving targets. Staring circular synthetic aperture radar (S-CSAR) provides unique opportunities for exploitation of radio frequency (RF) data collected over a large ground spot. The same phase history may be processed in different manners to generate simultaneous S-CSAR products such as 2$D$ Video SAR, coherent and non-coherent change detection (CCD and NCD), and ground moving target indication (GMTI). Advanced signal processing techniques can take advantage of the S-CSAR geometry to produce 3-D scene reconstructions. The ability to transmit, record and process large volumes of S-CSAR data, to create high fidelity exploitation products, in real-time, poses significant challenges. This paper addresses several open problems in this research area.
\end{abstract}

\section{INTRODUCTION}

Air-to-ground radars have traditionally been developed as narrow beam, scanning systems. These systems have significant constraints such as low revisit rates, low power efficiency, low resolution, and difficult sensor management problems. Also, since scanning systems are pre-tasked to perform specific functions, forensic analysis of old data is often impossible.

Staring circular synthetic aperture radar (SCSAR) offers significant improvements in exploitation functions by continually staring at an area of interest (AOI) with a very wide beam. The sensor platform flies a circular flight path at a constant elevation around a fixed scene center, and the AOI is constantly radiated with SAR-like pulses. This type of system largely overcomes the limitations of the traditional air-to-ground approach.

The primary benefit of S-CSAR systems is that several different functions can be performed simultaneously with the same radio frequency $(\mathrm{RF})$ data, such as imaging, stationary target detection, ground moving target indication (GMTI), and change detection. Notably, the choice of data product can be made after the collection, thus simplifying sensor management and allowing for forensic analysis.

Several challenges exist in terms of generating

*Sensors Directorate, US Air Force Research Laboratory, 2241 Avionics Circle, Building 620, Wright-Patterson AFB, $\mathrm{OH} 45433$, e-mail: linda.moore2@wpafb.af.mil high fidelity exploitation products from S-CSAR data collected over large ground spots. Challenge S-CSAR data sets and several open problems in the research areas of Video SAR, change detection, GMTI and 3-D scene reconstruction are offered in this paper.

\section{CHALLENGE DATA SETS}

AFRL has produced three carefully documented challenge problems for use by the larger research community. The challenge problems focus on 2D/3-D image reconstruction [1]; SAR GMTI [2]; and change detection and lossy phase history compression [3].

\section{VIDEO SAR}

Video SAR is a sequence of SAR images of the entire AOI produced at a given resolution and frame rate. Each SAR image is formed to the same pixel locations on the ground, and the height of each pixel could be derived from a Digital Elevation Model (DEM). Through this process, the images must be orthorectified so that the scene does not appear to rotate as the platform flies its circular path. Since it takes several seconds to form a synthetic aperture to achieve equivalent resolution in cross-range as that provided via bandwidth in range, successive frames of Video SAR have overlapped apertures.

Backprojection (BP), see [4] for a tutorial and sample code, was selected over the more computationally efficient Polar Format Algorithm (PFA) to leverage its inherent flexibility and pulse-wise processing, which allows intermediate results to be reused when processing overlapping apertures. To overcome the order $N^{3}$ computational requirements of $\mathrm{BP}$ and achieve real-time video SAR, fast BP algorithms have been developed $[5,6]$.

Improved computational efficiency for Video SAR remains an open research problem. S-CSAR introduces several processing challenges for an image formation processor (IFP). The relatively large scene invalidates a widely used plane wave approximation, which results in defocused and distorted images if the range curvature is not addressed. The IFP must also account for varying terrain and the non-linear circular flight path. Also, the overlapped

\section{U.S. Government work not protected by U.S. copyright}


apertures in successive frames of the Video SAR present an opportunity for re-use of calculations from previous frames. Finally, the IFP needs to maintain phase coherence across the image to enable more advanced processing described later in this paper.

\section{CHANGE DETECTION}

Change Detection (CD) analyzes two (or more) images of the same scene from the same aspect angle collected at different times to detect targets that have entered or exited the scene. A wide range of algorithms have been developed to address CD problems across numerous applications domains [7], including SAR, e.g., [8-10]. Since SAR data is complex-valued, encoding phase information, both coherent (CCD) [11] and non-coherent (NCD) operating modes may be considered. CCD offers the ability to detect extremely subtle changes in the scene, perhaps even at sub-wavelength scales, but requires careful preprocessing and image registration to obtain meaningful results. NCD offers coarser change resolution, but is simpler to implement and potentially more robust.

In general, $\mathrm{CD}$ offers several challenges for research. One primary concern is the registration of images from successive passes. In addition, even small variations in the flight path can produce significant variations in the strength and phase of returns, particularly from specular reflections, complicating their inter-pass cancellation. Furthermore, some target classes, like foliage, naturally decorrelate over time, producing undesired change detections. The following subsections describe several specific research thrusts dealing with $\mathrm{CD}$ in the context of S-CSAR.

\subsection{N-pass CD}

S-CSAR produces a stream of images of the AOI. Thus, multiple previous passes might be used to generate a more representative model of the scene for comparison, and new passes could be used to detect changes as well as to update this model. Noncausal processing of a sequence of passes could also be considered, akin to smoothing filtering operations. Over time, detailed statistics about the temporal properties of various clutter regions could also be collected, providing priors and informing confidence estimation when assessing changes in regions covered in foliage or similar features. Preliminary results in this direction, along with a Generalized Likelihood Ratio Test formulation for several scenarios, are provided in [12], but several open problems remain.

\subsection{Phase History Compression}

Possible avenues in pursuit of new SAR image formation algorithms include the development of phase history compression techniques, possibly through use of change detection from multiple passes. These phase history compression algorithms could reduce the amount of data to be processed from pass to pass by determining which portions of the phase history do not yield new valuable information. Two perspectives might be adopted when considering SAR data compression and CD. First, as described in [13], traditional image compression may lead to poor performance of CD, particularly CCD. Thus, new algorithms are required to efficiently compress SAR images without sacrificing CD performance. Similarly, the very existence of CD algorithms highlights the high degree of similarity between successive passes in a S-CSAR system. A lossy compression scheme could be developed to reduce the required data storage for new passes by encoding variations from a reference pass.

\subsection{CD for GMTI}

One of the key challenges in GMTI is the cancellation of clutter returns which vastly exceed the target amplitude. However, given previous passes from a S-CSAR system, an initial CCD step could drastically reduce the amplitude of clutter returns. The most basic incarnation of this idea is the clutter map used in traditional ground based MTI radars [14]. These rotating radars store a model of the clutter return as a function of look angle and range as the state of a stable infinite impulse response (IIR) filter which is continually updated with the new scan as an input.

A S-CSAR system possesses a conceptually similar map of the clutter for cancellation. Furthermore, this idea can be combined with N-pass CCD to produce a robust clutter map along with statistical information about clutter temporal correlation properties. See [15-18] for preliminary results along these lines.

\section{SAR-BASED GMTI}

Even employing state-of-the-art Space Time Adaptive Processing (STAP) approaches, traditional GMTI systems can only detect targets above a given Minimum Discernible Velocity (MDV). One possible solution is SAR Moving Reference Processing (SAR-MRP) $[15,19,20]$, which essentially match filters the SAR data with the phase history corresponding to a moving scatterer rather than a stationary point. When combined with CCD and a 
scheme for intelligently selecting motion hypotheses from prior knowledge, this approach offers the potential for detecting targets with $0 \mathrm{MDV}$ from single channel SAR data, albeit at high computational cost. The following subsections describe several S-CSAR specific research thrusts.

\subsection{Motion Estimation}

SAR-MRP assumes constant acceleration of the targets of interest. In practice, targets will likely exhibit more complex maneuvering. Methods must be developed to estimate target motion efficiently, rather than relying on a brute-force search over target motion profiles. However, for the special case of linear motion for both target and SAR platform, [19] showed that all moving targets can be optimally imaged by adding a third dimension corresponding to cross range velocity. For imaging, orientation estimation may also be needed, see e.g., [21].

An open problem of interest is the detection and tracking of rotating targets, such as vehicles turning corners. SAR-MRP assumes the target is translating only.

In addition, when considering urban environments, numerous moving targets may appear together with similar position and velocity over an extended period, see e.g., [22]. Tracking algorithms, potentially leveraging target features, must be developed to disambiguate these clustered maneuvering targets. In addition, methods for track re-association after obscuration in urban environments must also be developed.

\subsection{Incorporation of Prior Knowledge}

S-CSAR has the potential to leverage extensive prior knowledge about the AOI from both previous passes and external sources. Beyond CD, techniques must also be explored for incorporating external knowledge sources including road networks, satellite imagery, laser radar maps of buildings and terrain, textual information, weather reports, and similar data [23]. As a motivating example, road networks can be used to break ambiguities in target locations, predict future target motion, and to anticipate target obscurations.

\section{3-D IMAGING}

A benefit of S-CSAR is the ability to coherently process very large synthetic apertures for enhanced resolution on stationary targets [24]. In particular, the significance of forming very large apertures is that resolution is limited by the beamwidth of individual scatterers [25]. Integration of data through- out an entire circular orbit or from orbit-to-orbit provides 3-D information [26].

Advanced signal processing techniques may be used to produce 3 -D reconstructions of the AOI, and could enable automatic DEM generation. These techniques include $3-\mathrm{D}$ image reconstruction using multiple passes by taking advantage of modified flight path configurations or interferometric SAR processing techniques, and tomographic imaging with a single pass.

\subsection{3-D Imaging with Multiple Passes}

The circular flight path (CFP) of S-CSAR can be modified to enable 3-D resolution. Multiple passes can create cylindrical and spiral flight paths which can be coherently processed into a 3-D image.

Interferometric SAR (IFSAR) processing allows for height estimation of reflected objects in a scene, and thus 3-D scene reconstruction, through use of at least two passes that are identical in azimuth extent, but differ in elevation. For S-CSAR, higherresolution $N$-pass IFSAR techniques $[27,28]$ are of particular interest.

\subsection{3-D Tomographic Imaging with a Single Pass}

A single CFP supports 3-D tomographic SAR image processing by exploiting the multiple aspects that are obtained as the radar circles the AOI [29]. This type of processing combines all of the data from a full orbit of the CFP to resolve and locate all scatterers in three spatial dimensions. The result is a single $3-\mathrm{D}$ image cube that represents a series of image slices that are uniformly distributed in height. Each slice of the image cube contains the 2-D image data that exists at the corresponding height. The impact of scatterers from other heights is greatly diminished.

\section{CONCLUSIONS}

This paper provides an overview of several open research problems in the area of S-CSAR signal processing. S-CSAR offers many benefits including the ability to simultaneously generate exploitation products such as Video SAR, CD, GMTI and 3-D imagery. Because the collected raw phase history is preserved, forensic analysis may be performed. In addition to the challenges in algorithm development discussed here, a S-CSAR system presents high performance computing (HPC) challenges given the large amount of data acquired. In summary, SCSAR inherently offers a diverse, rich set of technical challenges and problems to be addressed in the future by the radar community. 


\section{References}

[1] Curtis H. Casteel, Jr., L. A. Gorham, M. J. Minardi, S. M. Scarborough, K. D. Naidu, and U. K. Majumder, "A challenge problem for 2D/3D imaging of targets from a volumetric data set in an urban environment," E. G. Zelnio and F. D. Garber, Eds., vol. 6568, no. 1. SPIE, 2007, p. 65680D.

[2] S. M. Scarborough, Curtis H. Casteel, Jr., L. Gorham, M. J. Minardi, U. K. Majumder, M. G. Judge, E. Zelnio, M. Bryant, H. Nichols, and D. Page, "A challenge problem for SAR-based GMTI in urban environments," E. G. Zelnio and F. D. Garber, Eds., vol. 7337, no. 1. SPIE, 2009, p. 73370G.

[3] S. M. Scarborough, L. Gorham, M. J. Minardi, U. K. Majumder, M. G. Judge, L. Moore, L. Novak, S. Jaroszewksi, L. Spoldi, and A. Pieramico, "A challenge problem for SAR change detection and data compression," E. G. Zelnio and F. D. Garber, Eds., vol. 7699, no. 1. SPIE, 2010, p. 76990 U.

[4] L. A. Gorham and L. J. Moore, "SAR image formation toolbox for MATLAB," E. G. Zelnio and F. D. Garber, Eds., vol. 7699, no. 1. SPIE, 2010, p. 769906.

[5] A. F. Yegulalp, "Fast backprojection algorithm for synthetic aperture radar." IEEE, 1999, pp. 60-65.

[6] L. A. Gorham, U. K. Majumder, P. Buxa, M. J. Backues, and A. C. Lindgren, "Implementation and analysis of a fast backprojection algorithm," E. G. Zelnio and F. D. Garber, Eds., vol. 6237, no. 1. SPIE, 2006, p. 62370G.

[7] R. Radke, S. Andra, O. Al-Kofahi, and B. Roysam, "Image change detection algorithms: a systematic survey," Image Processing, IEEE Transactions on, vol. 14, no. 3, pp. 294-307, 2005.

[8] K. Ranney and M. Soumekh, "Adaptive change detection in coherent and noncoherent SAR imagery," in Radar Conference, 2005 IEEE International. IEEE, 2005, pp. 195-200.

[9] E. Rignot and J. van Zyl, "Change detection techniques for ERS-1 SAR data," Geoscience and Remote Sensing, IEEE Transactions on, vol. 31, no. 4, pp. 896-906, 1993.

[10] R. White, "Change detection in SAR imagery," International Journal of remote sensing, vol. 12, no. 2, pp. 339-360, 1991.

[11] M. Preiss, "Coherent Change Detection: Theoretical Description and Experimental Results," Defence Science and Technology Orginisation, Australia, Tech. Rep. DSTO-TR-1851, AR No. 013-634, 2006.

[12] L. M. Novak, "Change detection for multi-polarization multi-pass SAR (Invited Paper)," E. G. Zelnio and F. D. Garber, Eds., vol. 5808, no. 1. SPIE, 2005, pp. $234-246$.

[13] L. M. Novak and C. E. Frost, "Effects of SAR image compression on coherent change detection," E. G. Zelnio and F. D. Garber, Eds., vol. 7337, no. 1. SPIE, 2009, p. 73370 S.

[14] M. Skolnik et al., RADAR Handbook. McGraw Hill, 2008.

[15] S. Scarborough, C. Lemanski, H. Nichols, G. Owirka, M. Minardi, and T. Hale, "SAR change detection MTI," E. G. Zelnio and F. D. Garber, Eds., vol. 6237, no. 1. SPIE, 2006, p. $62370 \mathrm{~V}$.
[16] D. Page and G. Owirka, "Knowledge-aided STAP processing for ground moving target indication radar using multilook data," EURASIP Journal on Applied Signal Processing, vol. 8, 2006.

[17] N. Goodman and P. Gurram, "STAP training through knowledge-aided predictive modeling," in Proceedings of the IEEE Radar Conference, 2004, pp. 388-393.

[18] G. A. Showman and W. L. Melvin, "Multi-resolution processing to enhance knowledge-aided STAP," in Proceedings 2003 DARPA/AFRL KASSPER Workshop, 2003.

[19] M. J. Minardi, L. A. Gorham, and E. G. Zelnio, "Ground moving target detection and tracking based on generalized SAR processing and change detection (Invited Paper)," E. G. Zelnio and F. D. Garber, Eds., vol. 5808, no. 1. SPIE, 2005, pp. 156-165.

[20] M. E. Holston, M. A. Temple, M. A. Saville, and M. J. Minardi, "Characterizing geolocation ambiguity responses in synthetic aperture radar: ground moving target indication," E. G. Zelnio and F. D. Garber, Eds., vol. 6568. SPIE, 2007.

[21] M. Ferrara and G. Arnold, "Shape and motion estimation from near-field echo-based sensor data," SIAM Journal on Imaging Sciences, vol. 2, no. 2, pp. 710-729, 2009.

[22] W. Melvin, R. Hancock, M. Rangaswamy, and J. Parker, "Adaptive distributed radar," in Radar Conference - Surveillance for a Safer World, 2009. RADAR. International, oct. 2009, pp. $1-6$.

[23] J. Guerci and E. Baranoski, "Knowledge-aided adaptive radar at DARPA: an overview," Signal Processing Magazine, IEEE, vol. 23, no. 1, pp. 41-50, 2006.

[24] R. L. Moses, L. C. Potter, and M. Cetin, "Wide Angle SAR Imaging," E. G. Zelnio and F. D. Garber, Eds., vol. 5427. SPIE, 2004.

[25] L. J. Moore and L. C. Potter, "Three-dimensional resolution for circular synthetic aperture radar," E. G. Zelnio and F. D. Garber, Eds., vol. 6568, no. 1. SPIE, 2007, p. 656804.

[26] E. Ertin, C. D. Austin, S. Sharma, R. L. Moses, and L. C. Potter, "Gotcha Experience Report: ThreeDimensional SAR Imaging with Complete Circular Apertures," E. G. Zelnio and F. D. Garber, Eds., vol. 6568. SPIE, 2007.

[27] C. D. Austin, E. Ertin, and R. L. Moses, "Sparse Multipass 3D SAR Imaging: Applications to the Gotcha Data Set," E. G. Zelnio and F. D. Garber, Eds., vol. 7337. SPIE, 2009.

[28] F. Lee-Elkin, "Autofocus for 3D Imaging," E. G. Zelnio and F. D. Garber, Eds., vol. 6970. SPIE, 2008.

[29] Charles V. Jakowatz, Jr., D. E. Wahl, P. H. Eichel, D. C. Ghiglia, and P. A. Thompson, Spotlight-Mode Synthetic Aperture Radar: A Signal Processing Approach. New York, N.Y.: Springer Science+Business Media, Inc., 1996. 